\title{
Augmented reality in the surgery of cerebral arteriovenous malformations: technique assessment and considerations
}

\author{
Ivan Cabrilo • Philippe Bijlenga • Karl Schaller
}

Received: 17 June 2014 / Accepted: 10 July 2014 / Published online: 20 July 2014

(C) Springer-Verlag Wien 2014

\begin{abstract}
Background Augmented reality technology has been used for intraoperative image guidance through the overlay of virtual images, from preoperative imaging studies, onto the realworld surgical field. Although setups based on augmented reality have been used for various neurosurgical pathologies, very few cases have been reported for the surgery of arteriovenous malformations (AVM). We present our experience with AVM surgery using a system designed for image injection of virtual images into the operating microscope's eyepiece, and discuss why augmented reality may be less appealing in this form of surgery.

Methods $N=5$ patients underwent AVM resection assisted by augmented reality. Virtual three-dimensional models of patients' heads, skulls, AVM nidi, and feeder and drainage vessels were selectively segmented and injected into the microscope's eyepiece for intraoperative image guidance, and their usefulness was assessed in each case.

Results Although the setup helped in performing tailored craniotomies, in guiding dissection and in localizing drainage veins, it did not provide the surgeon with useful information concerning feeder arteries, due to the complexity of AVM angioarchitecture.

Conclusion The difficulty in intraoperatively conveying useful information on feeder vessels may make augmented reality a less engaging tool in this form of surgery, and might explain its underrepresentation in the literature. Integrating an AVM's hemodynamic characteristics into the augmented rendering could make it more suited to AVM surgery.
\end{abstract}

I. Cabrilo $(\bowtie) \cdot$ P. Bijlenga $\cdot$ K. Schaller

Neurosurgery Division, Department of Clinical Neurosciences,

Faculty of Medicine, Geneva University Medical Center, Rue

Gabrielle-Perret-Gentil 4, 1211 Genève 14, Switzerland

e-mail: ivan.cabrilo@hcuge.ch
Keywords AVM surgery $\cdot$ Augmented reality $\cdot$ Image guidance $\cdot$ Minimal invasiveness $\cdot$ Neuronavigation

\section{Introduction}

There is a growing interest in the neurosurgical community for alternative, more intuitive intraoperative image guidance systems. Traditional neuronavigation, although arguably one the most useful tools at the neurosurgeon's disposal [15], is limited by the fact that it is point-based, relying on the use of a bayonet probe or electromagnetic stylet that translates information from the three-dimensional (3-D) real-world environment of the surgical field to two-dimensional (2-D) sections on the neuronavigation screen $[5,19]$. The surgeon is, therefore, confronted with the mental task of integrating these two image data sets.

Augmented reality technology, based on the overlay of virtual images on real-world structures [17], has the potential to solve this problem. Reports of variable setups applying augmented reality to a variety of neurosurgical pathologies have come out in recent years $[1-3,5-8,11-14,20]$. To date, a PubMed search for the terms "augmented reality neurosurgery" yields 55 results, of which 13 were published since 2013, and five so far for the year 2014 alone. However, only two results appear when one searches for "augmented reality arteriovenous malformation": A seminal article from 1999 presenting an augmented reality setup based on image injection into the operating microscope [10], and an article describing the development of a prototype system intended for arteriovenous malformation (AVM) surgery [9].

We have developed a standard operating procedure based on augmented reality with image injection directly into the operating microscope's eyepiece [2] and have applied this setup to a prospective series of patients with AVMs. Here we present our experience with AVM surgery assisted by 
augmented reality neuronavigation, and discuss its advantages as well as the encountered setbacks specific to this pathology, perhaps accounting for its underrepresentation in the literature.

\section{Methods}

The procedure used here has been detailed in a previous publication [2]. We briefly describe it with particular emphasis given to the specificities of AVM surgery. Informed patient consent was obtained.

Patients and setup assessment

From December 2012 to April 2014, $n=5$ patients underwent surgery for resection of AVMs assisted by augmented reality, in a hybrid neurointerventional suite equipped with flat-panel display technology (Allura Xper FD20; Philips, Best, the Netherlands), allowing for intraoperative 3-D digital subtraction angiography (DSA) for resection control.

The usefulness of augmented reality was evaluated in each case through four Boolean parameters: Whether the virtual images had an impact on the size and shape of the craniotomy; whether the virtual images helped in minimizing dissection; whether the virtual images helped in identifying the feeder arteries; and whether they helped in identifying the draining veins.

\section{Instrumentation}

Patients were positioned on the operating table and their heads fixed in a radiolucent head-holder (Mayfield; Integra LifeScience, Plainsboro, NJ, USA). Patients were registered to the neuronavigation station (Kolibri; BrainLAB, Feldkirchen, Germany) using face surface-matching systems (Z-touch or Softouch, Kolibri; BrainLAB, Feldkirchen, Germany), relative to the reference star fixed to the headholder. A separate, dedicated reference star was fixed to the microscope, and the microscope was then calibrated to the patient's neuronavigation reference star.

\section{Methodology}

3-D models of patients' heads and skulls, and of AVMs' nidi, feeder arteries, and draining veins, were preoperatively segmented in a single 3-D matrix (Fig. 2b), from 3-D image data sets [3-D DSA (Fig. 2a), angio-magnetic resonance imaging (angio-MRI), angio-computed tomography (angio-CT)], using the Iplan platform (BrainLAB, Feldkirchen, Germany).

These segmentations were intraoperatively injected into the microscope's eyepiece after microscope calibration was performed. First, 3-D models of patients' heads were injected to evaluate visually the accuracy of neuronavigation coregistration, through the degree of superposition between the patients' virtual heads and their real heads (Fig. 1a-c). If mismatch was encountered, the microscope was recalibrated on the reference star, and the accuracy of the neuronavigational data was re-assessed.

Once this was judged to be accurate, selective image injection of the AVM's components (nidus, feeder arteries, and drainage veins) was performed before draping (Fig. 2c-d), to plan the incision and to anticipate the angle of surgery, and, if necessary, to optimize the position of the patient's head by turning it in one piece, together with the head-holder and reference star. After draping and after making an incision along the planned line, the accuracy of neuronavigation was re-assessed using bony features. Image injection of the AVM and of the skull was used to tailor the craniotomy (Fig. 2e-f). After dural opening, the injected images of the AVM's components were used to guide dissection, localize the nidus, and identify the feeder arteries and the drainage veins (Fig. 2g).

\section{Results}

Patients, pathology, and postoperative outcome

Patient and AVM characteristics are summarized in Table 1. Patient 1 was pregnant at the time of AVM rupture and when she underwent surgery; intraoperative angiography was, therefore, not performed in her case. Total AVM resection was confirmed in her case after postoperative angiography at 1 year, which is standard protocol in our institution for all patients, despite complete resection indicated by intraoperative angiography. Follow-up is ongoing for patients $2-5$.

\section{Usefulness of augmented reality}

Results are summarized in Table 1 . In patient 1 , the hematoma from rupture was still present at the time of surgery, and immediate preoperative MRI, although clearly indicative of an arteriovenous anomaly, could not determine with precision the AVM's constituents. Segmentation of these structures could, therefore, not be performed in detail, which is why image injection only helped with tailoring the craniotomy and could not truly guide dissection nor identify the implicated vessels. In all other patients, 3-D DSA was repeated once the hematoma had resorbed to allow precise analysis of the AVM's angioarchitecture, so as to adopt the best therapeutic strategy for each patient. 


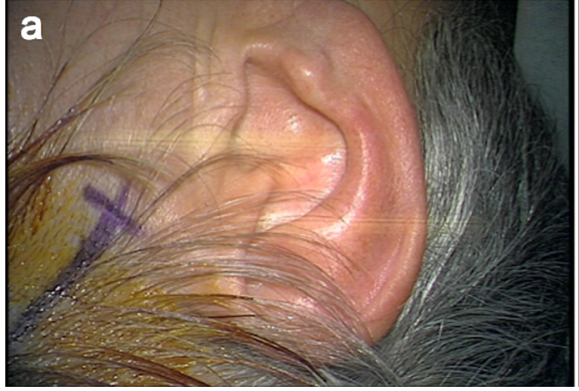

Fig. 1 Image injection, into the microscope's eyepiece, of the patient's head allows evaluation of the precision of neuronavigation. Here, the 3-D overlay of a patient's right ear is shown to illustrate this. Patients' facial
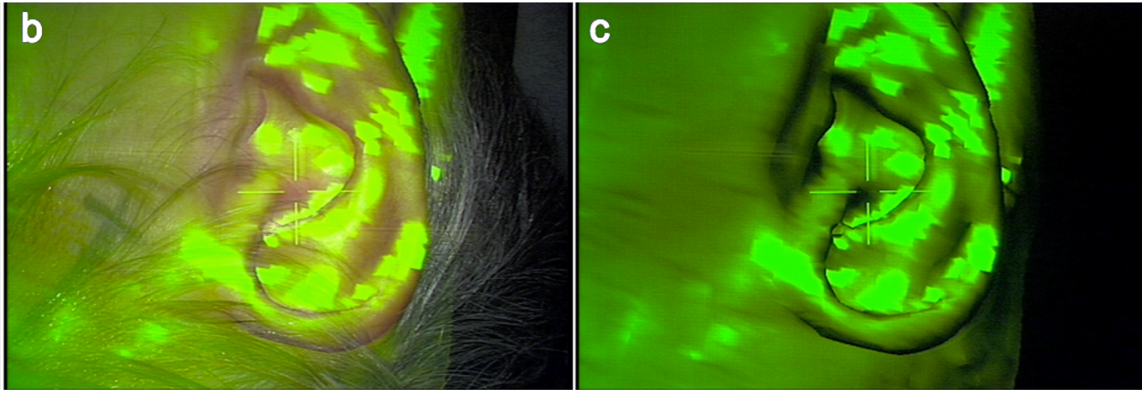

features can also be used for this purpose. Note how the virtual images accurately align with the real-world auricular structures. The intensity of image injection can be increased a-c or decreased

\section{Discussion}

To our knowledge only two reports of augmented realityguided AVM resections exist in the literature: Kockro et al. used a setup that augments video stream from a handheld video probe, for the resection of a right cerebellar AVM [11]; and King et al. illustrate their system, a prototype for image injection into the microscope's eyepiece [4], through the resection of a $2-\mathrm{cm}$ parietal AVM [10]. The same case is revisited in a subsequent publication from this group [5].

The setup we use is based on the system proposed by $[4,5$, 10], where virtual segmentations from preoperative imaging studies (angio-MRI, angio-CT, 3-D DSA) are injected into the eyepiece of the operating microscope (Figs. 1b-c and 2c-g). As the microscope's optics are also navigated, the system integrates the microscope's spatial, focus, and zoom parameters so that the augmented overlay appears at all times in accordance to all of these, relative to the navigated patient. This is in contrast to other setups, where image alignment with the surgical field is performed manually $[6,12,14]$. Furthermore, the presented system overlays augmented images directly onto the surgeon's field of vision and does not require having to look away to a separate screen, as is the case in previous reports $[3,7,11,13]$. Although an augmented video screen could be beneficial for endoscopic procedures [8] or for pre-incisional planning [3], our setup makes use of material that would probably have been used in any event for AVM surgery: The microscope and, in cases of deep-seated lesions, the neuronavigation station; no other hardware is required. Lastly, the setup allows 3-D stereoscopic visualization of the augmented field.

This last point is an important asset for AVM surgery, where the entangled vessels often result in confusing conformations, but it is not in itself sufficient for depth perception. Although not yet reported to have been applied to clinical practice, Kersten-Oertel et al. have developed a prototype image-guidance system intended for AVM surgery, where they specifically address the issue of depth perception [9]. The authors explore two visualization techniques: Colorcoding, where different colors are assigned to a head phantom's nidus, feeder and drainage vessels; chromadepth, where a color is assigned to a level of depth. However, although intuitively better suited, the latter technique did not convey a good perception of depth. Of note, the augmented display in this report was monoscopic.

Our setup integrates both visualization modalities. A color is selected during the preoperative segmentation of an anatomical structure, and during intraoperative image injection, this color-code is respected. Furthermore, stereoscopic image injection of volume-rendered 3-D models of the segmented structures conveys a sense of relative depth (Fig. 2e-g). Chromadepth is integrated through the fact that two different levels of transparency in the segmented models attempt to bestow a sense of depth between what is above and below the point of focus (Fig. 2e and g). Hence, depth perception of an augmented model is maximal when dissection has reached the structure in question. Adjusting the intensity of image injection, so as to accommodate the conflicting virtual images and real-world environment, can further this experience (Fig. 1ac). Finally, moving the microscope adds a parallax cue to depth perception.

However, in our clinical experience, the limiting factor in applying augmented reality to AVM surgery is not perception of depth, but rather, and more simply, the actual process of segmenting useful anatomical structures for the purpose of intraoperative guidance.

As Yasargil put it to depict the complexity in the relations between an AVM and its feeders [21]: "As any given AVM can derive its feeding arteries from a single artery or a number of arteries there are over 60 possible combinations of feeding vessels for that lesion." Furthermore, arteries may be terminal feeders, transit (partial) feeders, or en passage non-feeders. And distinguishing between these types is often still performed during surgery, despite advances in angiographic diagnostics. Therefore, while one or two easy-to-segment veins constitute an AVM's drainage system, segmenting the feeders can be an altogether difficult task.

This is illustrated by our results (Table 1), where augmented reality was used in only one - relatively straightforward case for feeder vessel identification; with the exception of 

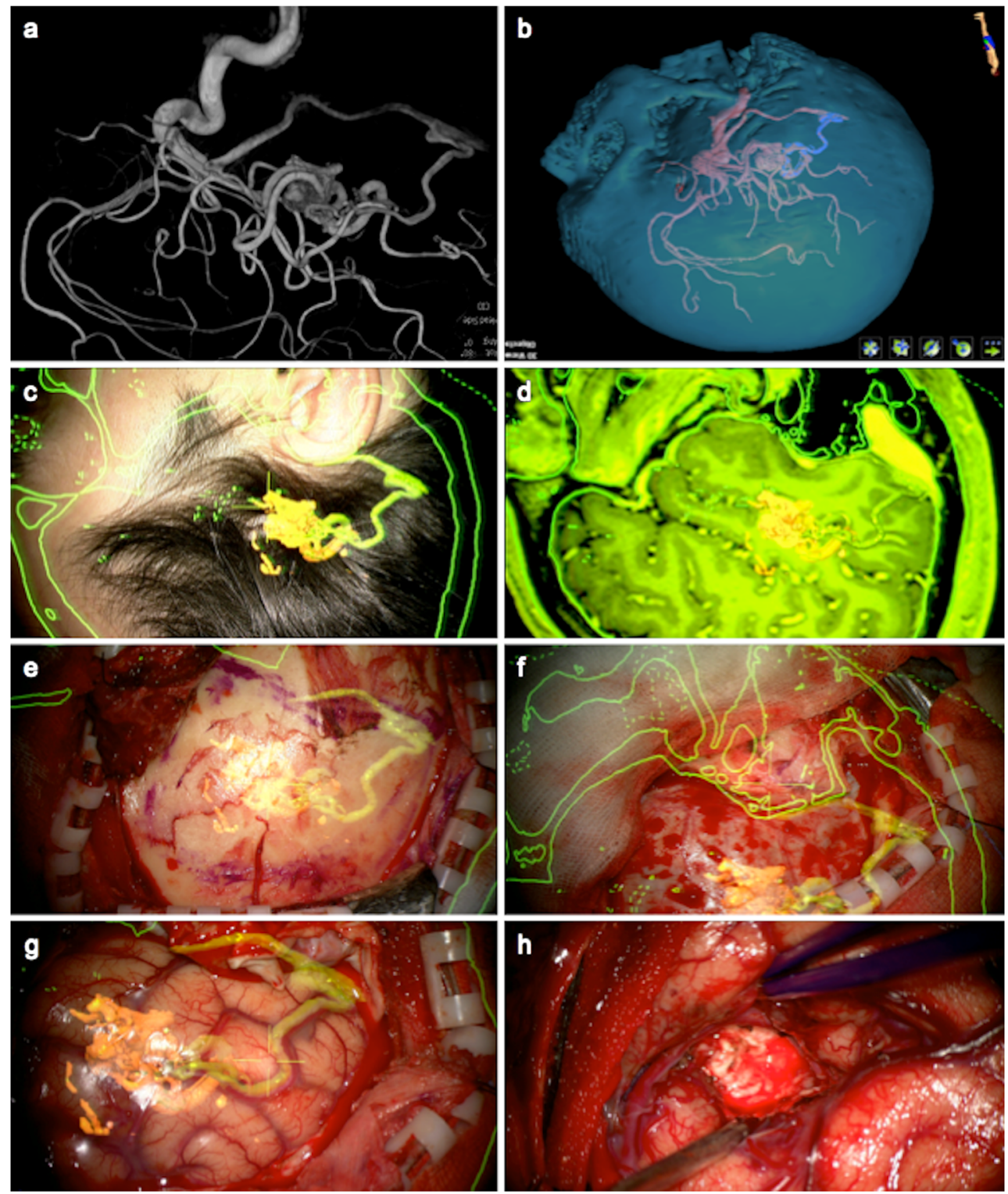

Fig. 2 This figure illustrates Case 5 in Table 1. a Preoperative 3-D DSA of a right temporal AVM, located in the depth of the superior temporal sulcus, and fed by branches of the middle cerebral artery, with a large vein draining into the transverse sinus at the junction with the sigmoid sinus. Reflux into the superior petrosal sinus can also be observed. The DSA has been turned to match the surgeon's view during the operation. b Segmentation of the patient's skull, vessels, and AVM, where the draining vein appears in blue. The segmentation has been turned to match the surgeon's view. c Image injection, onto the surface of the patient's head, of the AVM's nidus, and draining vein. This allows the surgeon to plan the incision. Note also image injection in 2-D of the patient's skull. d

patient 1, where the hematoma impeded adequate feeder vessel segmentation, image injection of feeder vessels was not useful in the other cases due to the complexity of the vascular bundle. Nevertheless, image injection of the main feeder trunk was useful in that it indicated with precision where proximal control should be performed in case of periprocedural AVM rupture. The usefulness of image injection in identifying the

Image injection, also into the microscope's oculars, for general orientation of the patient's MRI along with the segmented AVM and its draining vein, clearly seen draining into the transverse-sigmoid sinus junction. e After incision, image injection of the AVM and of underlying bony structures helps to plan the craniotomy performed in $\mathbf{f}$. Note how the craniotomy is perfectly aligned to the underlying petrous bone, in the supramastoid region. $\mathbf{g}$ After dural opening, the AVM's draining vein is clearly identified through image injection of the virtual draining vein. The virtual image of the AVM indicates with precision where the real AVM is buried, and thereby guides dissection. $\mathbf{h}$ After total AVM resection

drainage vessels also seems to be disappointingly low; however, this is because certain cases were embolized, allowing greater freedom for intraoperative exploration, explaining why this augmented information was not necessary. In these cases, the role of image injection was very much like that in tumor resection: In effect, augmented reality was useful in all cases for tailoring the craniotomy and for guided dissection, 


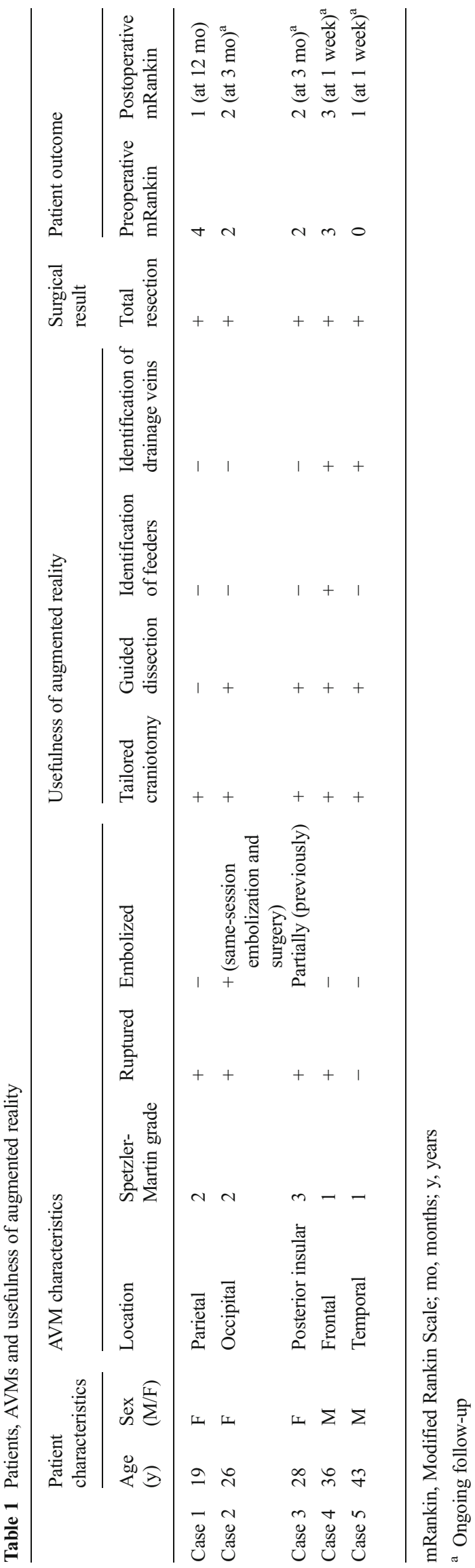

with the exception of patient 1 for the already mentioned reason.

The interest behind augmented reality is being able to provide a selective and simplified representation of anatomy, readily understood during surgery. This is exemplified by its use during tumor $[3,5,7,8,11-13]$ or cavernoma surgery [11, 20] where it helps in performing targeted craniotomies and dissections; in aneurysm surgery, where it helps in intraoperatively appreciating the aneurysm's angioarchitecture [2]; or in extracranial-to-intracranial bypass surgery, where it can help in localizing donor and recipient vessels and performing mini-craniotomies. Consequently, the difficulty in offering a simplified representation of an AVM's constituents might contribute to making this technique less appealing to groups using augmented reality.

The underlying question is whether this technology can nevertheless bring an added value during AVM surgery. As mentioned earlier, one main advantage of our setup is that it makes use of material that would have in any case been in the operating theater. But is it truly efficient to use, for the sake of tailoring a craniotomy, as it might only be in certain cases? On the basis of the idea that any information helping to achieve the surgical goal is valuable (Stadie AT, Comment to [2]), and that, intuitively, smaller craniotomies are associated to fewer complications $[16,18]$, we would venture that the setup, as it is presented here, is worthwhile.

Of note, in certain cases, although not encountered in this patient series, the venous drainage can involve transosseous diploic veins or dural veins; augmenting the surgical field with this information can be very useful during craniotomy and durotomy to avoid potentially disastrous consequences at the very beginning of surgery.

Nonetheless, besides clear anatomical information for intraoperative orientation regarding feeder vessels and the regional anatomy of nidi, it would be interesting for future development to integrate hemodynamic information into the augmented surgical field, derived from preoperative angiography. In other words, in the absence of clearly demarcated feeder vessels, virtual 4-D hemodynamic color-coded information could provide the surgeon with regional information of the AVM and what nature of vessel he or she is likely to encounter.

\section{Conclusion}

Although the difficulty in helping the surgeon localize an AVM's feeder vessels remains an obstacle, the presented setup, based on image injection into the operating microscope's eyepiece, can be successfully integrated into AVM surgical procedures. It can help in tailoring the craniotomy to the individual patient's anatomy, in guiding dissection and in identifying draining veins. Integrating an AVM's hemodynamic 
information could make the system better suited to this particular surgery.

Conflicts of interest The authors declare that they have no conflict of interest.

\section{References}

1. Birkfellner W, Figl M, Huber K, Watzinger F, Wanschitz F, Hummel J, Hanel R, Greimel W, Homolka P, Ewers R, Bergmann H (2002) A head-mounted operating binocular for augmented reality visualization in medicine-design and initial evaluation. IEEE Trans Med Imaging 21:991-997

2. Cabrilo I, Bijlenga P, Schaller K (2014) Augmented reality in the surgery of cerebral aneurysms: a technical report. Neurosurgery 10(Suppl 2):252-261

3. Deng W, Li F, Wang M, Song Z (2014) Easy-to-use augmented reality neuronavigation using a wireless tablet PC. Stereotact Funct Neurosurg 92:17-24

4. Edwards PJ, Hawkes DJ, Hill DL, Jewell D, Spink R, Strong A, Gleeson M (1995) Augmentation of reality using an operating microscope for otolaryngology and neurosurgical guidance. J Image Guid Surg 1:172-178

5. Edwards PJ, King AP, Maurer CR Jr, de Cunha DA, Hawkes DJ, Hill DL, Gaston RP, Fenlon MR, Jusczyzck A, Strong AJ, Chandler CL, Gleeson MJ (2000) Design and evaluation of a system for microscope-assisted guided interventions (MAGI). IEEE Trans Med Imaging 19:1082-1093

6. Gleason PL, Kikinis R, Altobelli D, Wells W, Alexander E 3rd, Black PM, Jolesz F (1994) Video registration virtual reality for nonlinkage stereotactic surgery. Stereotact Funct Neurosurg 63:139-143

7. Inoue D, Cho B, Mori M, Kikkawa Y, Amano T, Nakamizo A, Yoshimoto K, Mizoguchi M, Tomikawa M, Hong J, Hashizume M, Sasaki T (2013) Preliminary study on the clinical application of augmented reality neuronavigation. J Neurol Surg A Cent Eur Neurosurg 74:71-76

8. Kawamata T, Iseki H, Shibasaki T, Hori T (2002) Endoscopic augmented reality navigation system for endonasal transsphenoidal surgery to treat pituitary tumors: technical note. Neurosurgery 50:13931397
9. Kersten-Oertel M, Chen SS, Drouin S, Sinclair DS, Collins DL (2012) Augmented reality visualization for guidance in neurovascular surgery. Stud Health Technol Inform 173:225-229

10. King AP, Edwards PJ, Maurer CR Jr, de Cunha DA, Hawkes DJ, Hill DL, Gaston RP, Fenlon MR, Strong AJ, Chandler CL, Richards A, Gleeson MJ (1999) A system for microscope-assisted guided interventions. Stereotact Funct Neurosurg 72:107-111

11. Kockro RA, Tsai YT, Ng I, Hwang P, Zhu C, Agusanto K, Hong LX, Serra L (2009) Dex-ray: augmented reality neurosurgical navigation with a handheld video probe. Neurosurgery 65:795-807

12. Lovo EE, Quintana JC, Puebla MC, Torrealba G, Santos JL, Lira IH, Tagle P (2007) A novel, inexpensive method of image coregistration for applications in image-guided surgery using augmented reality. Neurosurgery 60:366-371

13. Low D, Lee CK, Dip LL, Ng WH, Ang BT, Ng I (2010) Augmented reality neurosurgical planning and navigation for surgical excision of parasagittal, falcine and convexity meningiomas. Br J Neurosurg 24 : 69-74

14. Mahvash M, Besharati Tabrizi L (2013) A novel augmented reality system of image projection for image-guided neurosurgery. Acta Neurochir 155:943-947

15. Mert A, Gan LS, Knosp E, Sutherland GR, Wolfsberger S (2013) Advanced cranial navigation. Neurosurgery 72(Suppl 1):43-53

16. Paleologos TS, Wadley JP, Kitchen ND, Thomas DG (2000) Clinical utility and cost-effectiveness of interactive image-guided craniotomy: clinical comparison between conventional and image-guided meningioma surgery. Neurosurgery 47:40-47, discussion 47-48

17. Peters TM (2006) Image-guidance for surgical procedures. Phys Med Biol 51:R505-540

18. Radovanovic I, Abou-Hamden A, Bacigaluppi S, Tymianski M (2014) A safety, length of stay, and cost analysis of minimally invasive microsurgery for anterior circulation aneurysms. Acta Neurochir 156:493-503

19. Shuhaiber JH (2004) Augmented reality in surgery. Arch Surg 139: 170-174

20. Stadie AT, Reisch R, Kockro RA, Fischer G, Schwandt E, Boor S, Stoeter P (2009) Minimally invasive cerebral cavernoma surgery using keyhole approaches - solutions for technique-related limitations. Minim Invasive Neurosurg 52:9-16

21. Yasargil MG (1987) Pathological Considerations: Elements of an AVM. Microneurosurgery. vol IIIA: AVM of the Brain, History, Embryology, Pathological Considerations, Hemodynamics, Daignostic Studies, Microsurgical Anatomy. Thieme New York, pp $111-138$ 